\title{
Cellular localization and putative role of aquaporin-2 Ser-261 in the bovine kidney
}

\author{
K. Michałek ${ }^{1,3}$, M. Grabowska² and A. Lepczyński ${ }^{1}$ \\ ${ }^{1}$ West Pomeranian University of Technology in Szczecin, Faculty of Biotechnology and Animal Husbandry, \\ Department of Physiology, Cytobiology and Proteomics, Klemensa Janickiego 29, 71-270 Szczecin, Poland \\ ${ }^{2}$ Pomeranian Medical University, Department of Histology and Developmental Biology, Żołnierska 48, 71-210 Szczecin, Poland
}

KEY WORDS: aquaporin, phosphorylation, kidneys, immunohistochemistry, cattle

Received: $\quad 7$ June 2018

Revised: 27 November 2018

Accepted: 18 February 2019

\begin{abstract}
Aquaporin-2 (AQP2) is a small, transmembrane protein, active in the principal cells of the renal collecting duct system. Phosphorylation and dephosphorylation of this protein regulate its redistribution in the cell, thereby influencing renal water reabsorption. The aims of the study were to identify and analyse the location of AQP2 phosphorylated at position 261, and to determine its potential role in renal water balance regulation, because data on AQP2 in cattle are scarce, and the mechanisms regulating its expression and cellular localization remain unclear. The analyses were carried out on kidneys collected from eight Polish Holstein-Friesian Black-and-White male calves, aged 5-7 months. It was found, using immunohistochemistry and commercially available phosphospecific antibodies, that in cattle AQP2 Ser-261 is located exclusively in the apical plasma membrane of the principal cells of the collecting duct. This atypical location of AQP2 Ser-261 allows to conclude that it may play a significant role in the process of renal water retention. The results of our previous studies on the regulation of renal total AQP2 excretion with urine in calves, as well as the bioinformatic analysis of available data presented in this study, seem to support this assumption. In addition, bioinformatic tools predicted mitogen activated protein kinases (MAPK) as a possible vasopressin-independent factor involved in AQP2 Ser-261 phosphorylation and accumulation in the apical plasma membrane.
\end{abstract}

${ }^{1}$ Corresponding author:

e-mail: kmichalek@zut.edu.pl in the apical membrane and intracellular vesicles of renal collecting duct principal cells (Michałek et al., 2014b). We have also confirmed in our studies on calves that AQP2 in cattle is excreted in urine, similarly as in other mammals. However, the mechanisms regulating renal excretion of this protein, especially during the postnatal period, seem to be slightly different in humans and laboratory animals. In fact, the observed increase in AQP2 excretion in calves in response to additional water losses along with faeces in the course of diarrhoea was not accompanied by a typical increase in plasma vasopressin (AVP) concentration (Michałek et al., 2014a). 
A number of studies have been published in recent years, specifying factors that possibly regulate its expression and cellular localization, due to the great interest in AQP2, especially in the context of using its urine level as a potential biomarker of renal dysfunctions (Brown et al., 2008; Fenton et al., 2008; Holmes, 2012; Kwon et al., 2013). Among them, the works on intracellular signalling pathways, and the sites and role of AQP2 phosphorylation gained our particular interest. It has been found that there are other pathways involved in the regulation of $\mathrm{AQP} 2$ expression and localization, in addition to AVP and its activated intracellular pathway associated with adenyl cyclase (AC) cAMP - protein kinase A (PKA) (Moeller et al., 2011; Moeller and Fenton, 2012). PKA, activated by vasopressin and increased cAMP concentration, causes AQP2 phosphorylation in its $\mathrm{COOH}$-terminal tail at serine residue 256 (Ser-256), which leads to its accumulation in the apical plasma membrane of the collecting duct principal cells (Fushimi et al., 1997; Nishimoto et al., 1999). Bioinformatics data analyses have shown that AQP2 contains many other putative recognition sequences, which are target sites for many various kinases, including mitogen activated protein kinases (MAPK), protein kinase $\mathrm{G}$ (PKG), casein kinase II or glycogen synthase kinase 3 (van Balkom et al., 2002; Brown, 2008; Brown et al., 2008; Rieg et al., 2010; Moeller et al., 2011). However, currently available experimental data include four phosphorylated serines: Ser-256, Ser-261, Ser-264 and Ser-269, which are located within the last 16 amino acids of the AQP2 $\mathrm{COOH}$ terminus. Studies on experimental animal models and in vitro cell cultures clearly demonstrated that serine phosphorylation and dephosphorylation at these sites affected AQP2 redistribution in cells and, as a consequence, their water permeability (Brown et al., 2008).

In the available literature there is lack of complete data on the phosphorylation sites of bovine AQP2 and their phosphorylating kinases. We conducted a bioinformatic analysis of data concerning potential phosphorylation sites of AQP2 in cattle in reference to the results of our previous studies, indicating the participation of other AVP-independent factors causing an increase of this protein urinary excretion in calves. The use of bioinformatics tools allowed the highest probability prediction of AQP2 phosphorylation at Ser-256 and Ser-261 sites. As mentioned earlier, the phosphorylation of AQP2 Ser-256 is mainly associated with AVP stimulation. Since then we were seeking an explanation for other alternative, AVP-independent bovine mechanisms regulating renal water excretion involving AQP2, we decided to identify and precisely localize phosphorylated AQP2 at Ser-261 in kidneys of young beef cattle and select potential kinases involved in its regulation. On the basis of the results and collected data, the subsequent aim of the work was to suggest the potential role of AQP2 Ser-261 in the process of renal water retention in cattle.

\section{Material and methods}

\section{Animals and immunohistochemistry}

We analysed 8 kidneys from 8 Polish Holstein-Friesian Black-and-White male calves, aged 5-7 months, obtained from a local abattoir. Immediately after slaughter, the kidneys were rapidly removed, fixed in $4 \%$ buffered paraformaldehyde, embedded in paraffin block, and sliced into $2 \mu \mathrm{m}$-thick sections on a rotary microtome (MICROM HM 340, MICROM Laborgeräte, Walldorf, Germany).

Immunohistochemical reaction was performed according to the protocol previously described in detail by Michałek et al. (2014b). To reveal antigens, sections were incubated in $1 \mathrm{mM}$ Tris solution ( $\mathrm{pH}$ 9.0) supplemented with 0.5 mM EGTA and heated in a microwave oven for $10 \mathrm{~min}$. Non-specific Ig binding was prevented by incubating the sections in $50 \mathrm{mM} \mathrm{NH}_{4} \mathrm{Cl}$ for $30 \mathrm{~min}$, followed by blocking in PBS supplemented with $1 \%$ BSA, $0.05 \%$ saponin and $0.2 \%$ gelatin. The sections were incubated overnight at $4{ }^{\circ} \mathrm{C}$ with primary antibodies diluted in the $1: 100$ ratio in $0.01 \mathrm{M}$ PBS with $0.1 \% \mathrm{BSA}$ and Triton X-100. The sections were rinsed with $0.01 \mathrm{M}$ PBS and incubated for $1 \mathrm{~h}$ at room temperature with horseradish peroxidase-conjugated anti-rabbit secondary antibodies diluted in the 1:500 ratio (BIORAD STAR124P, Hercules, California, USA). An immunohistochemical reaction of 3.3'-diaminobenzidine in chromogen solution (K3468, Dako, Glostrup, Denmark) was used for visualization. Mayer's haematoxylin was used as a counterstain. The sections were examined using a optical microscope (Olympus BX 41, Olympus, Hamburg Germany). The specificity of immunostaining was confirmed by following the above procedures in the absence of the primary antibody (negative control; Figure 1F).

In the present study, a rabbit anti-AQP2 (pSer261) polyclonal primary antibody (AHP1299, BIO-RAD, Hercules, California, USA) was used to examine the detailed cellular location of AQP2 Ser-261 in the kidneys of the tested animals. 


\section{Bioinformatic analysis}

The analysis involved in creating a graphical model of bovine AQP2 was based on the data available in the UniProt database (www.uniprot.org) for the protein, accession number P79099. An analysis was performed using a ModPred (Indiana University, Bloomington, Indiana, USA) bioinformatic software package in order to determine possible serine residue phosphorylation sites and the probability of such modifications. Thereafter, using the available bioinformatics tools: NetPhos (Technical University of Denmark, Copenhagen, Denmark), KinasePhos (National Chiao Tung University, Hsinchu, Taiwan), ELM (ELM Consorptium, Heidelberg, Germany) and NetPhorest (University of Copenhagen, Copenhagen, Denmark), amino acid sequences characteristic for phosphorylation sites were predicted as well as protein kinases that may be responsible for the phosphorylation of the indicated serine residues.

\section{Results}

Immunolocalization of AQP2 Ser-261 in the kidney of young beef cattle is shown in Figure 1.AQP2 Ser-26 was found in the collecting ducts of medullary rays that penetrate the renal cortex (Figure $1 \mathrm{~A}-\mathrm{C}$ ) and in the collecting ducts of the renal medulla (Figure 1D-E). Strong expression of AQP2 Ser-261 was exclusively visible in the apical plasma membrane of the principal cells of the collecting duct. There was no visible immunohistochemical reaction in the intracellular vesicles and the basolateral membrane.

The general probability of bovine AQP2 protein phosphorylation sites was predicted based on bioinformatic analysis using the ModPred bioinformatics tool. The results clearly showed the highest phosphorylation possibility of Ser-256 and Ser-261 residues and moderate and minor probability of phosphorylation of Ser-264 and Ser-269 residues.
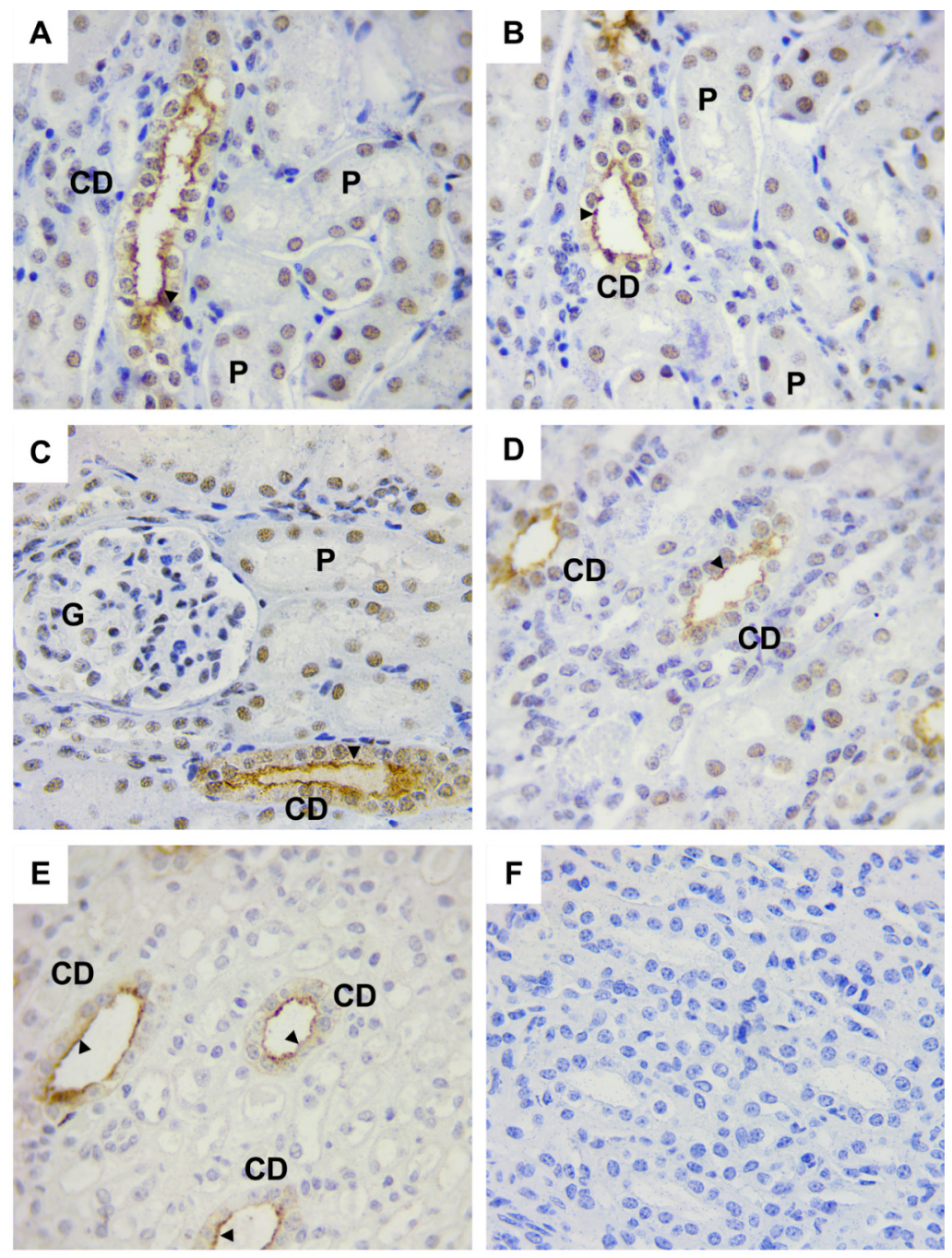

Figure 1. Immunolocalization of aquaporin-2 (AQP2) Ser-261 in the renal cortex (A, B and C) and renal medulla (D and E) of male calves AQP2 Ser-261 is detected in the apical plasma (black arrowhead) of medullary collecting duct principal cells; $P$ - proximal tubule, $G$ - glomerulus, $\mathrm{D}$ - distal tubule, $\mathrm{CD}$ - collecting duct; $\mathrm{F}$ - negative control of immunohistochemical reaction; magnification: $\times 600$ 


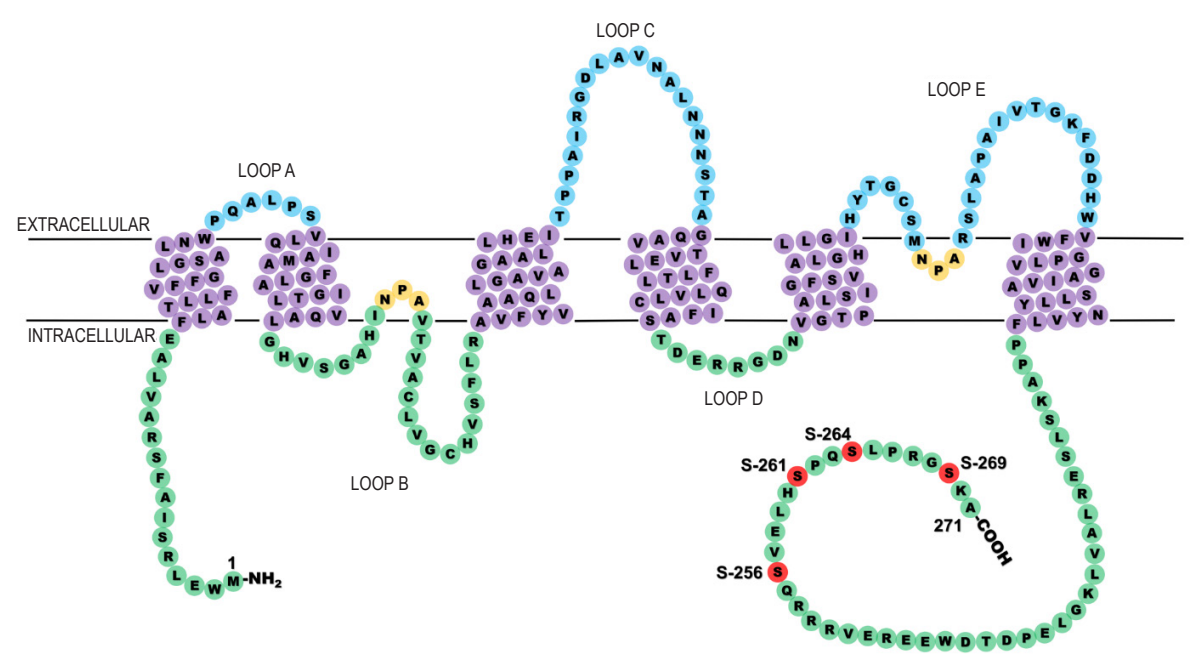

Figure 2. The full-length amino acid sequence of bovine aquaporin-2 monomer (P79099) and putative phosphorylation sites (based on UniProt, www.uniprot.org)

The amino acid sequences of the possible phosphorylation sites with bolded serine residue are shown in Table 1. Potential phosphorylation sites are also depicted on the bovine AQP2 monomer model presented in Figure 2. Subsequently, putative kinases for mentioned serine residues (Ser-256, Ser-261, Ser-264 and Ser-269) were determined using various bioinformatics tools, which are listed in Table 1.

Table 1. Predicted kinases involved in the phosphorylation of serine residues: Ser-256, Ser-261, Ser-264 and Ser-269 of bovine aquaporin-2 (AQP2)

\begin{tabular}{|c|c|c|c|}
\hline Serine & $\begin{array}{l}\text { General probability } \\
\text { of serine residue } \\
\text { phosphorylation }\end{array}$ & Kinase & $\begin{array}{l}\text { Phosporylation } \\
\text { site sequence }\end{array}$ \\
\hline Ser-256 & High & $\begin{array}{l}\text { PKAABCD } \\
\text { RSK }^{A} \\
\text { PKC } \\
\text { PKG } \\
\text { PKG } \\
\text { CLK }^{\mathrm{B}} \\
\text { DMPK }^{\mathrm{D}}\end{array}$ & RRRQSVELH \\
\hline Ser-261 & High & $\begin{array}{l}\text { MAPK } K^{A B C D} \\
\text { CDK5 }\end{array}$ & VELHSPQSL \\
\hline Ser-264 & Medium & $\begin{array}{l}\text { PKC }{ }^{A D} \\
\text { CK1 }^{A} \\
\text { CDK2 }^{A}\end{array}$ & HSPQSLPRG \\
\hline Ser-269 & Low & PKA ${ }^{A C D}$ & LPRGSKA-- \\
\hline
\end{tabular}

Prediction was established using different bioinformatic tools denoted by capital letters: A - NetPhos (Technical University of Denmark, Copenhagen, Denmark), ${ }^{\mathrm{B}}$ - KinasePhos (National Chiao Tung University, Hsinchu, Taiwan), ${ }^{\mathrm{C}}$ - ELM (ELM Consorptium, Heidelberg, Germany), ${ }^{D}$ - NetPhorest (University of Copenhagen, Copenhagen, Denmark). General probability of AQP2 serine phosphorylation was determined using the ModPred (Indiana University, Bloomington, Indiana, USA) bioinformatic software. Bold marks serine residues in the amino acid sequence as potential phosphorylation sites. Abbreviations: PKA - protein kinase A; RSK - $90 \mathrm{kDa}$ ribosomal S6 kinase; PKC - protein kinase C; PKG - protein kinase G; CLK - CDC like kinase; DMPK - myotonin-protein kinase; MAPK - mitogen activated protein kinase; CDK5 - cyclin dependent kinase 5; CK1 - cell kinase 1 group; CDK2 - cyclin dependent kinase 2.

\section{Discussion}

To our knowledge, this is the first report on the identification and cellular localization of AQP2 Ser-261 in cattle. Until now, no data have been published on renal expression of different phosphorylated forms of AQP2 and putative kinases. The only information on this subject is derived from the studies on laboratory animals and in vitro cell culture models. In this work, we have shown that AQP2 Ser-261 is expressed exclusively in the apical parts of the collecting duct principal cells of the bovine kidney. Phosphorylated serine in AQP2 at position 261 was not detected in the basolateral membrane and intracellular vesicles of these cells in the kidneys of the test animals.

It is widely known that AVP is the main regulatory factor of the abundance and cellular localization of AQP2 in mammals. In the absence of AVP stimulation, AQP2 is stored in intracellular vesicles of the connecting tubule (CT) and collecting duct principal cells (CCT). Vasopressin, synthesized in the hypothalamus, is released to the blood from the posterior pituitary gland in response to hypovolemia or hypernatremia (Kortenoeven and Fenton, 2014). AVP binds to vasopressin type 2 receptor (V2R), which is located in the basolateral membrane of renal CT and CCT (Wilson et al., 2013). Binding of this peptide hormone to V2R stimulates the Gs protein alpha submit, activates adenylate cyclases (AD), increases intracellular cyclic adenosine monophosphate (cAMP) production, activates protein kinases and elevates intracellular calcium levels. As a result, AQP2 undergoes phosphorylation, followed by its redistribution to the plasma membrane (Nejsum et al., 2005; Moeller et al., 2011). 
In vitro studies on the inner medullary collecting duct (IMCD) suspension and laboratory animals have shown that AQP2 phosphorylation at Ser256, Ser-264 and Ser-269 increased in response to dDAVP (desmopressin, V2R agonist) administration or cAMP addition (Hoffert et al., 2007, 2008; Fenton et al., 2008). Their redistribution in the cell also changes in response to AVP stimulation. AQP2 Ser256, Ser-264 and Ser-269 are then mainly located in the apical plasma membrane of the principal cells of the collecting duct (Fushimi et al., 1997; Christensen et al., 2000; Kamsteeg et al., 2000; Fenton et al., 2008; Takata et al., 2008; Moeller et al., 2009; Eto et al., 2010; Tamma et al., 2011). Phosphoproteomic analysis of the rat inner medullary collecting duct was one of the methods used to show that AQP2 phosphorylation at serine 261 decreased in response to AVP accumulation, which indicated its low involvement in AQP2 plasma membrane targeting (Hoffert et al., 2006; Tamma et al., 2014; Yui et al., 2017).

It has been also found that AQP2 Ser-261 is located in mice and rats in the endoplasmic reticulum, Golgi apparatus and lysosomes (Hoffert et al., 2007). In our study it was demonstrated that in contrast to laboratory animals, AQP2 Ser-261 in cattle is present in the apical plasma membrane of the principal cells of the collecting duct. Thus, the results indicate that the role of AQP2 Ser-261 in water transport into cells is probably significantly greater in cattle than in laboratory animals. Presumably, AQP2 Ser-261 in the bovine CCT is also involved in other processes occurring in cells. Namely, according to Moeller et al. (2011), in addition to the regulation of AQP2 distribution in the cell, phosphorylation may also affect channel gating, exocytosis, endocytosis, 'active structure' formation and degradation of AQP2. Phosphorylation of AQP2 is also involved in the regulation of cell volume recovery in response to hypotonic stress (Li et al., 2009).

The apparent, exclusive accumulation of AQP2 Ser-261 in the apical plasma membrane observed in the tested calves allowed assuming that this protein, phosphorylated at this position, is most likely primarily involved in water transport to the interior of principal cells and is removed to urine by exocytosis. This could partially explain the increase in renal AQP2 excretion in calves observed in our previous studies with a simultaneous lack of the increase in blood plasma AVP concentration (Michałek et al., 2014a). As mentioned before, AVP-dependent increase in cAMP production and PKA activation are the main factors responsible for the phosphorylation of AQP2 at serine position 256.
However, many putative protein kinases may be involved in this process (Table 1). As emphasized by Moeller et al. (2011), when determining the role of putative protein kinases in modulating channel function, in addition to sequence recognition, one should also include their presence in the cells of the same type as target proteins or their activation by certain factors. Based upon bioinformatic analysis, AQP2 Ser-261 phosphorylation in cattle is most probably regulated by MAPK and cyclin dependent kinase 5 (CDK5) (Table 1), which are both expressed in renal epithelial cells (Kiely et al., 2003; Guevara et al., 2014). However, their activating factors in cattle, leading to a potential phosphorylation of AQP2 Ser-261, accumulation in the apical membrane and probable subsequent exocytosis, are difficult to indicate at the moment. Tamma et al. (2014) found a significant impact of CDK activation and a new independent pathway regulating AQP2 trafficking on an increase in AQP2 Ser-261 phosphorylation. The authors found that the application of a specific CDK inhibitor ex vivo in the kidney slice and MDCK (Madin-Darby Canine Kidney) cells significantly reduced AQP2 Ser-261 levels. However, MAPK, which in rats is activated under acute hypertonicity (Hasler et al., 2008), may play an important role in trafficking and accumulation of AQP2 in the plasma membrane in cattle. In the absence of AVP stimulation, hypertonicity alone induced rapid plasma membrane AQP2 accumulation in rat kidney CCT (Hasler et al., 2008). Perhaps it is MAPK activation that is the main source of increased AQP2 accumulation in the apical membrane and its total excretion with urine in calves in response to additional water loss with faeces under osmotic diarrhoea (Michałek et al., 2014a). MAPK activation, which probably plays a significant role in the bovine kidney signalling cascade, may also explain the presence of AQP2 Ser-261 in the apical plasma membrane. Bioinformatic analyses of the data have demonstrated that it is fairly unlikely that MAPK phosphorylates AQP2 at position 256.

The results obtained in the study regarding the location and role of AQP2 Ser-261 in the kidneys of the tested animals undoubtedly provide new data in this field in cattle. Nevertheless, it should be emphasized that in view of still very limited data and numerous precariousnesses, it is necessary to conduct further, detailed research that would allow better understanding of kidney function and full explanation of the role of AQP2 phosphorylation in water balance regulation in cattle in the future. 


\section{Conclusions}

It was revealed that aquaporin-2 (AQP2) Ser-261 in the kidney of young beef cattle is localized exclusively in the apical plasma membrane of the principal cells in the collecting duct. The characteristic location of AQP2 phosphorylated at position 261 in this mammal indicates its significant role in the transport of water into the cells and, as a consequence, in renal water retention and concentrated urine production. Bioinformatic data analysis predicted mitogen activated protein kinases (MAPK) as a putative agent involved in AQP2 Ser-261 phosphorylation.

\section{Acknowledgments}

The work was supported by a scientific grant from the National Science Centre, Poland (Miniatura No. 2017/01/X/NZ9/00814).

\section{References}

Brown D., 2008. Aquaporins and vasopressin signaling in the kidney health and diseases: introduction. Semin. Nephrol. 28, 215-216, https://doi.org/10.1016/j.semnephrol.2008.03.001

Brown D., Hasler U., Nunes P., Bouley R., Lu H.A.J., 2008. Phosphorylation events and the modulation of aquaporin 2 cell surface expression. Curr. Opin. Nephrol. Hypertens. 17, 491-498, https:// doi.org/10.1097/MNH.0b013e3283094eb1

Christensen B.M., Zelenina M., Aperia A., Nielsen S., 2000. Localization and regulation of PKA-phosphorylated AQP2 in response to V(2)-receptor agonist/antagonist treatment. Am. J. Physiol. Renal Physiol. 278, F29-F42, https://doi.org/10.1152/ ajprenal.2000.278.1.F29

Eto K., Noda Y., Horikawa S., Uchida S., Sasaki S., 2010. Phosphorylation of aquaporin-2 regulates its water permeability. J. Biol. Chem. 285, 40777-40784, https://doi.org/10.1074/jbc. M110.151928

Fenton R.A., Moeller H.B., Hoffert J.D., Yu M.-J., Nielsen S., Knepper M.A., 2008. Acute regulation of aquaporin-2 phosphorylation at Ser-264 by vasopressin. Proc. Natl. Acad. Sci. USA 105, 3134-3139, https://doi.org/10.1073/pnas.0712338105

Fushimi K., Sasaki S., Marumo F., 1997. Phosphorylation of serine 256 is required for cAMP-dependent regulatory exocytosis of aquaporin-2 water channel. J. Biol. Chem. 271, 14800-14804, https://doi.org/10.1074/jbc.272.23.14800

Guevara T., Sancho M., Pérez-Payá E., Orzáez M., 2014. Role of CDK5/ cyclin complexes in ischemia-induced death and survival of renal tubular cells. Cell Cycle 13, 1671-1626, https://doi. org/10.4161/cc. 28628

Hasler U., Nunes P., Bouley R., Lu H.A.J., Matsuzaki T., Brown D., 2008. Acute hypertonicity alters aquaporin-2 trafficking and induces a MAPK-dependent accumulation at the plasma membrane of renal epithelial cells. J. Biol. Chem. 283, 26643-26661, https:// doi.org/10.1074/jbc.M801071200

Hoffert J.D., Fenton R.A., Moeller H.B., Simons B., Tchapyjnikov D., McDill B.W., Yu M.-J., Pisitkun T., Chen F., Knepper M.A., 2008. Vasopressin-stimulated increase in phosphorylation at $\mathrm{Ser}^{269}$ potentiates plasma membrane retention of aquaporin-2. J. Biol. Chem. 283, 24617-24627, https://doi.org/10.1074/jbc. M803074200
Hoffert J.D., Nielsen J., Yu M.-J., Pisitkun T., Schleicher S.M., Nielsen S., 2007. Dynamics of aquaporin-2 serine-261 phosphorylation in response to short-term vasopressin treatment in collecting duct. Am. J. Physiol. Renal Physiol. 292, F691-F700, https:/l doi.org/10.1152/ajprenal.00284.2006

Hoffert J.D., Pisitkun T., Wang G., Shen R.-F., Knepper M.A., 2006. Quantitative phosphoproteomics of vasopressin-sensitive renal cells: Regulation of aquaporin-2 phosphorylation at two sites. Proc. Natl. Acad. Sci. USA 103, 7159-7164, https://doi. org/10.1073/pnas.0600895103

Holmes R.P., 2012. The role of renal water channels in health and disease. Mol. Aspects Med. 33, 547-552, https://doi.org/10.1016/j. mam.2012.01.001

Kamsteeg E.J., Heijnen I., van Os C.H., Deen P.M.T., 2000. The subcellular localization of an aquaporin-2 tetramer depends on the stoichiometry of phosphorylated and nonphosphorylated monomers. J. Cell Biol. 151, 919-930, https://doi.org/10.1083/ jcb.151.4.919

Kiely B., Feldman G., Ryan M.P., 2003. Modulation of renal epithelial barrier function by mitogen-activated protein kinases (MAPKs): Mechanism of cyclosporine A-induced increase in transepithelial resistance. Kidney Int. 63, 908-916, https://doi.org/10.1046/ j.1523-1755.2003.00804.x

Kitchen P., Day R.E., Salman M.M., Conner M.T., Bill R.M., Conner A.C., 2015. Beyond water homeostasis: Diverse functional roles of mammalian aquaporins. Biochim. Biophys. Acta 1850, 2410-2421, https://doi.org/10.1016/j.bbagen.2015.08.023

Kortenoeven M.L.A., Fenton R.A., 2014. Renal aquaporins and water balance disorders. Biochim. Biophys. Acta 1840, 1533-1549, https://doi.org/10.1016/j.bbagen.2013.12.002

Kwon T.-H., Frøkiær J., Nielsen S., 2013. Regulation of aquaporin-2 in the kidney: A molecular mechanism of body-water homeostasis. Kidney Res. Clin. Pract. 32, 96-102, https://doi.org/10.1016/j. krcp.2013.07.005

Li Y.-H., Eto K., Horikawa S., Uchida S., Sasaki S., Li X.-J., Noda Y., 2009. Aquaporin-2 regulates cell volume recovery via tropomyosin. Int. J. Biochem. Cell Biol. 41, 2466-2476, https://doi. org/10.1016/j.biocel.2009.07.017

Michalek K., 2016. Aquaglyceroporin in the kidney: Present state of knowledge and prospects. J. Physiol. Pharmacol. 67, 185-193

Michałek K., Dratwa-Chałupnik A., Ciechanowicz A.K., Malinowski E., 2014a. Aquaporin 2: Identification and analysis of expression in calves' urine during their first month of life. Can. J. Anim. Sci. 94, 653-659, https://doi.org/10.4141/cjas-2014-023

Michałek K., Laszczyńska M., Ciechanowicz A.K., Herosimczyk A., Rotter I., Oganowska M., Lepczyński A., Dratwa-Chałupnik A., 2014b. Immunohistochemical identification of aquaporin 2 in the kidneys of young beef cattle. Biotech. Histochem. 89, 342-347, https://doi.org/10.3109/10520295.2013.858828

Moeller H.B., Fenton R.A., 2012. Cell biology of vasopressin-regulated aquaporin-2 trafficking. Pflugers. Arch. 464, 133-144, https:/l doi.org/10.1007/s00424-012-1129-4

Moeller H.B., Knepper M.A., Fenton R.A., 2009. Serine 269 phosphorylated aquaporin-2 is targeted to the apical membrane of collecting duct principal cells. Kidney Int. 75, 295-303, https://doi. org/10.1038/ki.2008.505

Moeller H.B., Olesen E.T.B., Fenton R.A., 2011. Regulation of the water channel aquapori-2 by psttranslation modification. Am. J. Physiol. Renal Physiol. 300, F1062-F1073, https://doi. org/10.1152/ajprenal.00721.2010

Nejsum L.N., Zelenina M., Aperia A., Frøkiær J., Nielsen S., 2005. Bidirectional regulation of AQP2 trafficking and recycling: involvement of AQP2-S256 phosphorylation. Am. J. Physiol. Renal Physiol. 288, F930-F938, https://doi.org/10.1152/ajprenal.00291.2004 
Nishimoto G., Zelenina M., Li D., Yasui M., Aperia A., Nielsen S., Nairn A.C., 1999. Arginine vasopressin stimulates phosphorylation of aquaporin-2 in rat renal tissue. Am. J. Physiol. 276, F254-F259, https://doi.org/10.1152/ajprenal.1999.276.2.F254

Rieg T., Tang T., Murray F., Schroth J., Insel P.A., Fenton R.A., Hammond H.K., Vallon V., 2010. Adenylate cyclase 6 determines CAMP formation and aquaporin-2 phosphorylation and trafficking in inner medulla. J. Am. Soc. Nephrol. 21, 2059-2068, https://doi.org/10.1681/ASN.2010040409

Takata K., Matsuzaki T., Tajika Y., Ablimit A., Hasegawa T., 2008. Localization and trafficking of aquaporin 2 in the kidney. Histochem. Cell. Biol. 130, 197-209, https://doi.org/10.1007/ s00418-008-0457-0

Tamma G., Lasorsa D., Trimpert Ch. et al., 2014. A protein kinase A-independent pathway controlling Aquaporin 2 trafficking as a possible cause for the syndrome of inappropriate antidiuresis associated with polycystic kidney disease 1 haploinsufficiency. J. Am. Soc. Nephrol. 25, 2241-2253, https://doi. org/10.1681/ASN.2013111234
Tamma G., Robben J.H., Trimpert Ch., Boone M., Deen P.M.T., 2011. Regulation of AQP2 localization by S256 and S261 phosphorylation and ubiquitination. Am J Physiol. Cell Physiol. 300, C636-C646, https://doi.org/10.1152/ajpcell.00433.2009

van Balkom B.W.M., Savelkoul P.J.M., Markovich D., Hofman E., Nielsen S., van der Sluijs P., Deen P.M.T., 2002. The role of putative phosphorylation sites in the targeting and shuttling of the aquaporin-2 water channel. J. Biol. Chem. 277, 41473-41479, https://doi.org/10.1074/jbc.M207525200

Wilson J.L.L., Miranda C.A., Knepper M.A., 2013. Vasopressin and the regulation of aquaporin-2. Clin. Exp. Nephrol. 17, 751-764, https://doi.org/10.1007/s10157-013-0789-5

Yui N., Sasaki S., Uchida S., 2017. Aquaporin-2 Ser-261 phosphorylation is regulated in combination with Ser-256 and Ser-269 phosphorylation. Biochem. Biophys. Res. Commun. 482, 524-529, https://doi.org/10.1016/j.bbrc.2016.11.118 